\title{
Recent results on hard probes in heavy-ion collisions from ALICE and LHCb
}

\author{
Ritsuya Hosokawa* for the ALICE and LHCb Collaborations \\ University of Tsukuba, Tsukuba, Japan \\ Université Grenoble Alpes, Grenoble, France \\ E-mail: r.hosokawadecern.ch
}

\begin{abstract}
The heavy-ion physics program at the ALICE and LHCb collaborations aims at studying the Cold Nuclear Matter (CNM) effects and revealing the properties of Quark-Gluon Plasma (QGP) which is hot and dense QCD matter consisting of deconfined quarks and gluons produced under extremely high energy density. Hard probes, originate from the initial hard scatterings with large momentum transfer, are excellent tools to study heavy-ion collisions. In this contribution, we report recent results on hard probes in heavy-ion collisions from the ALICE and LHCb collaborations.
\end{abstract}

7th Annual Conference on Large Hadron Collider Physics - LHCP2019

20-25 May, 2019

Puebla, Mexico

${ }^{*}$ Speaker. 


\section{Introduction}

Hard probes, such as jets and heavy flavours, are well calibrated probes, since they originate from initial hard scatterings with large momentum transfer $\left(Q^{2} \gg \Lambda_{Q C D}\right)$. They are calculable with perturbative Quantum ChromoDynamics (pQCD) and the final-state particle production in nucleonnucleon collisions is predictable with the factorization theorem [W]. The particle production in heavy-ion collisions is modified from the superposition of the binary nucleon-nucleon collisions due to several nuclear matter effects. Particularly, in high-energy heavy-ion collisions, the particle production is affected by several cold nuclear matter effects and effects from hot and dense QCD matter, known as Quark-Gluon Plasma (QGP). Experimentally, the cold nuclear matter effects, such as shadowing and anti-shadowing of the nuclear parton distribution functions, can be studied in small system collisions, such as nucleon-nucleus collisions, and the properties of QGP are studied in nucleus-nucleus collisions.

In this contribution, recent results on the measurements of hard probes from ALICE and LHCb experiments are reported.

\section{The ALICE and LHCb experiments}

The ALICE detector [ [ $]$ has been designed to study high energy heavy-ion collisions at the LHC. In ALICE, the measurements reported in this contribution are performed with the central barrel tracking detectors $(|\eta|<0.9)$, calorimeters $(|\eta|<0.7)$, and forward muon tracking system $(-4<\eta<-2.5)$. The LHCb detector [3] has been built to study the particles which contain $b$ or $c$ quarks (heavy-flavour). In the $\mathrm{LHCb}$, all detectors are instrumented in forward region $(2<\eta<5)$, which is unique coverage in comparison with the other experiments at the LHC. In addition, fixedtarget experiments using beam-gas interactions have been carried out by $\mathrm{LHCb}$ which are unique program at the LHC.

\section{Jets in $\mathbf{P b}-\mathbf{P b}$ collisions}

The nuclear modification factor of jets in the most central $\mathrm{Pb}-\mathrm{Pb}$ collisions at $\sqrt{s_{N N}}=5.02 \mathrm{TeV}$ as a function of transverse momentum is shown in the Fig. [ [ [ by factor 2 or more and is interpreted as the result of in-medium parton energy loss. The results are compared with several energy loss model predictions $[\square, \square, \square, \mathbb{Q}, \mathbf{Q}, \mathbf{Q}$. All models qualitatively describe the jet suppression but still there is a slight tension with the data quantitatively. Fig. shows a jet substructure measurement in the most central $\mathrm{Pb}-\mathrm{Pb}$ collisions at $\sqrt{s_{N N}}=2.76 \mathrm{TeV}$. Two hard prong substructure within jet cone is extracted by re-clustering and de-clustering with soft drop method [ए]]. The measured $z_{g}$, which is the fraction of leading and sub-leading prongs transverse momentum taken by sub-leading prong, is shown here. The selected distance between the two prongs, $\Delta R$, changes between the panels in Fig. $\square$. For larger $\Delta R$, suppression of the rate of symmetric splitting is found. A hint of enhancement of small angle splitting is also observed though it is not significant within current uncertainties. The results are compared to embedded PYTHIA pp reference and theoretical predictions [ [8, Q] 0 . All results are qualitatively described by the predictions. 

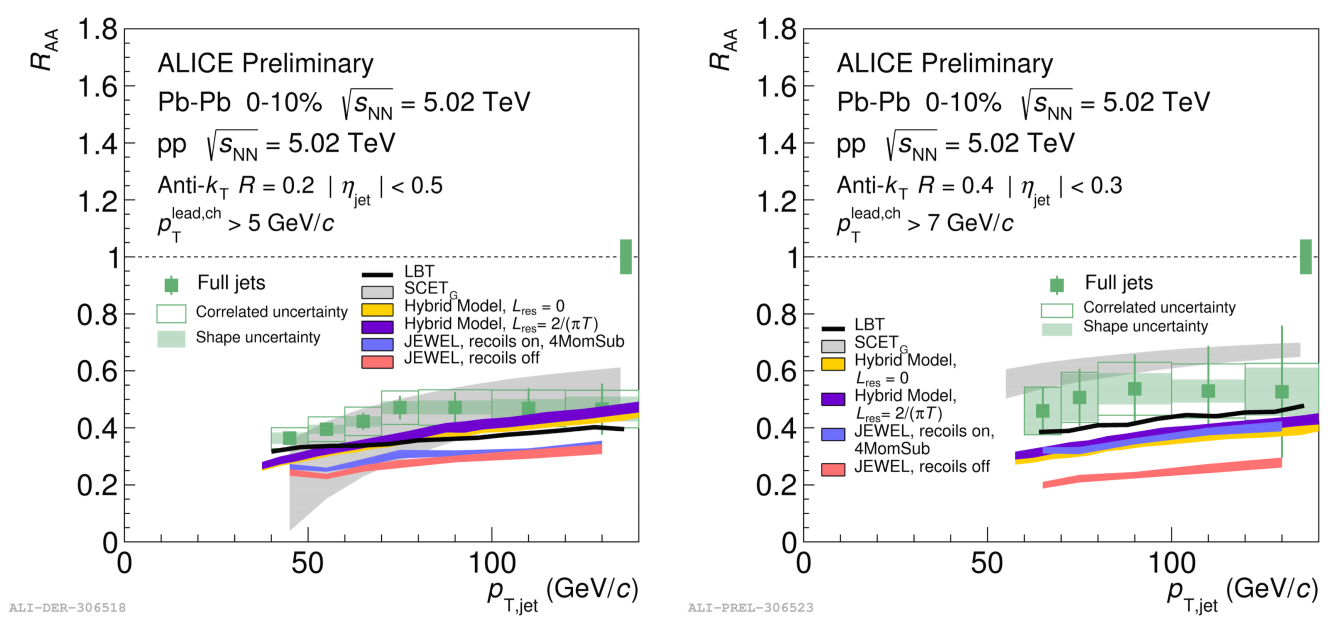

Figure 1: The nuclear modification factor of $R=0.2$ (left) and 0.4 (right) jets in $\mathrm{Pb}-\mathrm{Pb}$ collisions at $\sqrt{s_{N N}}$ $=5.02 \mathrm{TeV}$ [䧃]. The results are compared to LBT, SCETG, Hybrid model, and JEWEL predictions. The combined $\mathrm{T}_{A A}$ uncertainty and pp luminosity uncertainty is shown as a band on the dashed line at $R_{\mathrm{AA}}=1$.

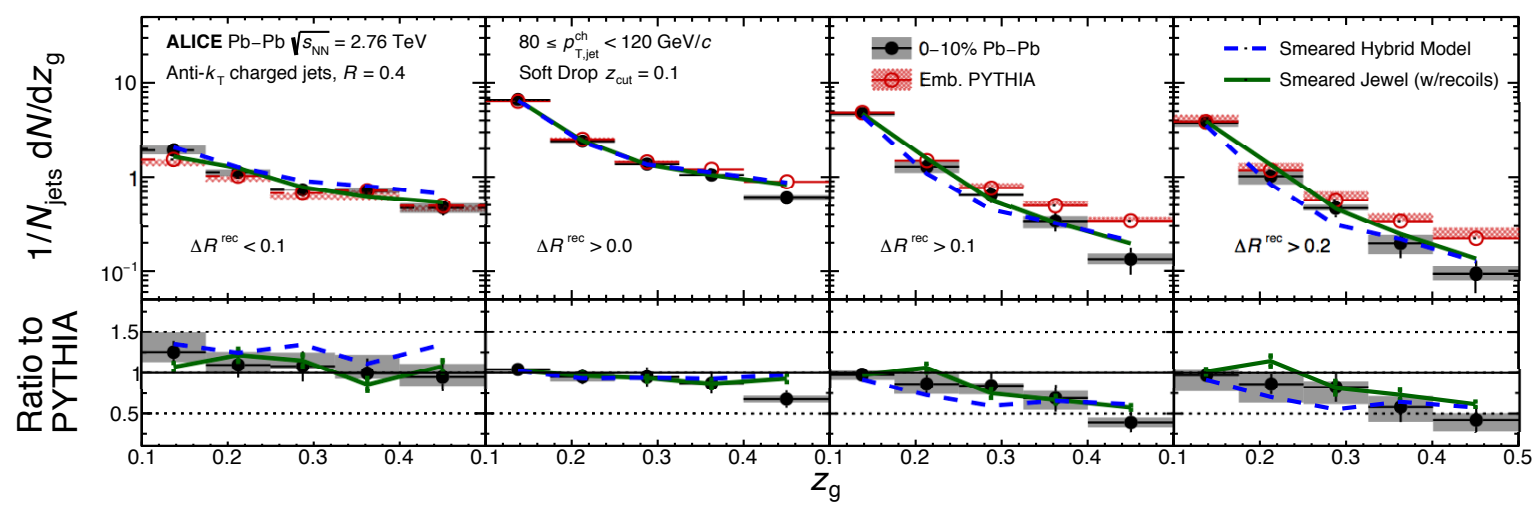

Figure 2: Detector-level distributions of $z_{g}$ for $R=0.4$ jets in $\mathrm{Pb}-\mathrm{Pb}$ collisions at $\sqrt{s_{N N}}=2.76 \mathrm{TeV}$ with varying minimum/maximum angular separation of subjets $(\Delta R)$ for jets in the range $80<p_{\mathrm{T}}^{c h}<120 \mathrm{GeV} / \mathrm{c}$ [ए]]. The shaded bands represent systematic uncertainties. The data results are compared with the embedded PYTHIA reference (open symbols), Hybrid model (dashed line) and JEWEL (solid line). The ratios of data, Hybrid and JEWEL model to the embedded PYTHIA reference are shown in the bottom panels.

\section{Open heavy flavour production in $\mathrm{Pb}-\mathrm{Pb}$ and $\mathrm{p}-\mathrm{Pb}$ collisions}

Fig. B (left) shows the nuclear modification factor of $\Lambda_{c}$ production as a function of $p_{\mathrm{T}}$ in $\mathrm{Pb}-$ $\mathrm{Pb}$ collisions at $\sqrt{s_{N N}}=5.02 \mathrm{TeV}[\mathbb{[}]$ and the result is compared to the production of $D$ meson and inclusive charged hadron in central collisions. The nuclear modification factor of charged hadrons, $D$ mesons, and $\Lambda_{c}$ shows clear ordering.

This result may be a hint of less suppression of $\Lambda_{c}$ production though the results are compared for different centrality classes. Fig. B (right) shows the production ratio of $\Lambda_{c}$ and $D^{0}$ meson in 

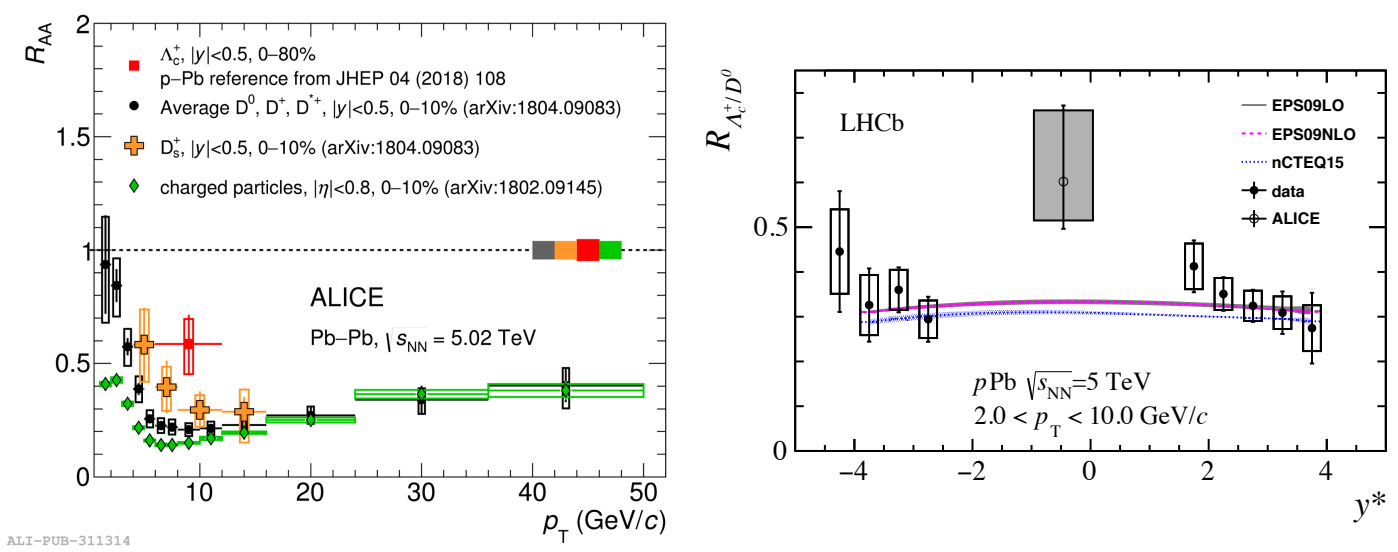

Figure 3: Left: The nuclear modification factor of $\Lambda_{c}^{+}$baryons as a function of $p_{\mathrm{T}}$ in $0-80 \% \mathrm{~Pb}-\mathrm{Pb}$ collisions at $\sqrt{s_{N N}}=5.02 \mathrm{TeV}[\mathbb{U}]$. The result is compared with the nuclear modification factors of D mesons (closed circle and cross) and charged hadrons (diamond). Normalisation uncertainties are represented by bands on the dashed line at $R_{\mathrm{AA}}=1$.

Right: The cross-section ratio between $\Lambda_{c}^{+}$baryons and $D^{0}$ mesons as a function of rapidity for $2<p_{\mathrm{T}}$ $<10 \mathrm{GeV} / \mathrm{c}$ range [ㅁ] $]$. The systematic uncertainty is represented by the boxes. The vertical error bar represents the quadratic sum of the statistical and the systematic uncertainties. The results are compared with the HELAC-Onia calculations with EPS09LO/NLO and nCTEQ15 nPDFs.

$\mathrm{p}-\mathrm{Pb}$ collisions at $\sqrt{s_{N N}}=5.02 \mathrm{TeV}$ [ए2] . The data at forward and backward rapidity range are described by the predictions with EPS09LO/NLO and nCTEQ15 nPDFs [13], प44, प5]]. On the other hand, discrepancy between the data and the predictions is observed at mid-rapidity. This result may be a hint of non-universality of the fragmentation functions. A rising trend as a function of rapidity is seen at forward rapidity. This trend may be compatible with the mid-rapidity result although the trend is not so significant within uncertainties.

\section{Quarkonia production in $\mathrm{Pb}-\mathrm{Pb}$ and $\mathrm{p}-\mathrm{Pb}$ collisions}

Fig. 1 shows the measurements of the elliptic flow coefficient $v_{2}$ of the $\Upsilon(1 \mathrm{~S})$ in $\mathrm{Pb}-\mathrm{Pb}$ collisions at $\sqrt{s_{N N}}=5.02 \mathrm{TeV}$ as a function of $p_{\mathrm{T}}$ and centrality [प]]. The $v_{2}(\Upsilon(1 S))$ are consistent with zero at all $p_{\mathrm{T}}$ and centrality bins within current uncertainties.

The $v_{2}$ of the $\Upsilon(1 \mathrm{~S})$ is smaller than that of $J / \psi$ with about $2 \sigma$ significance in 5-60\% and 20$60 \%$ centrality intervals. The results are compared with the predictions and all results are consistent with the predictions within uncertainties. Fig. $\square$ shows the result of $\Upsilon$ production for $1 \mathrm{~S}$ and $2 \mathrm{~S}$ states in $\mathrm{p}-\mathrm{Pb}$ collisions at $\sqrt{s_{N N}}=8.16 \mathrm{TeV}$ [ए耳]. Fig. $\mathrm{G}$ (left) is the nuclear modification factor of $\Upsilon(1 \mathrm{~S})$ and Fig. $[$ (right) is the one of $\Upsilon(2 S)$. It is found that the suppression is stronger for

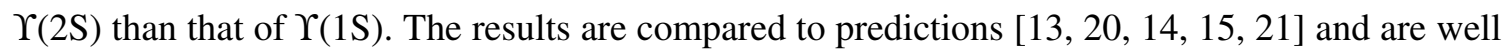
described by predictions with comover model.

\section{Charm production in $\mathbf{p}-\mathrm{He}$ and $\mathrm{p}-\mathrm{Ar}$ collisions with fixed target configuration}

The results of measurements of charm production in $\mathrm{p}-\mathrm{He}$ and $\mathrm{p}-\mathrm{Ar}$ collisions at $\sqrt{s_{N N}}=86.6$ 

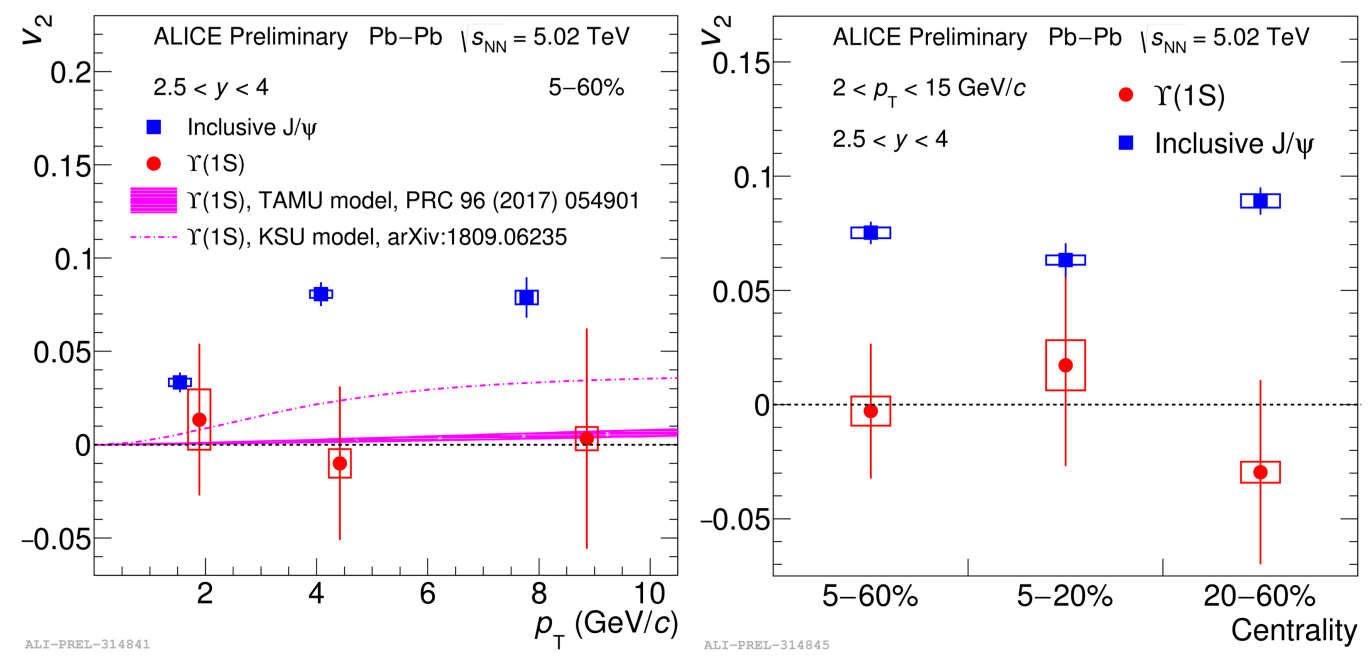

Figure 4: The elliptical flow coefficient $v_{2}$ of the $\Upsilon(1 \mathrm{~S})$ in $\mathrm{Pb}-\mathrm{Pb}$ collisions at $\sqrt{s_{N N}}=5.02 \mathrm{TeV}$ as a function of $p_{\mathrm{T}}$ (left) and centrality (right) [प] ]. The results are compared with the inclusive $J / \psi$. The results of TAMU model [ㅍ] calculation and KSU [[ष]] model calculation as a function of $p_{\mathrm{T}}$ are shown in the left figure.
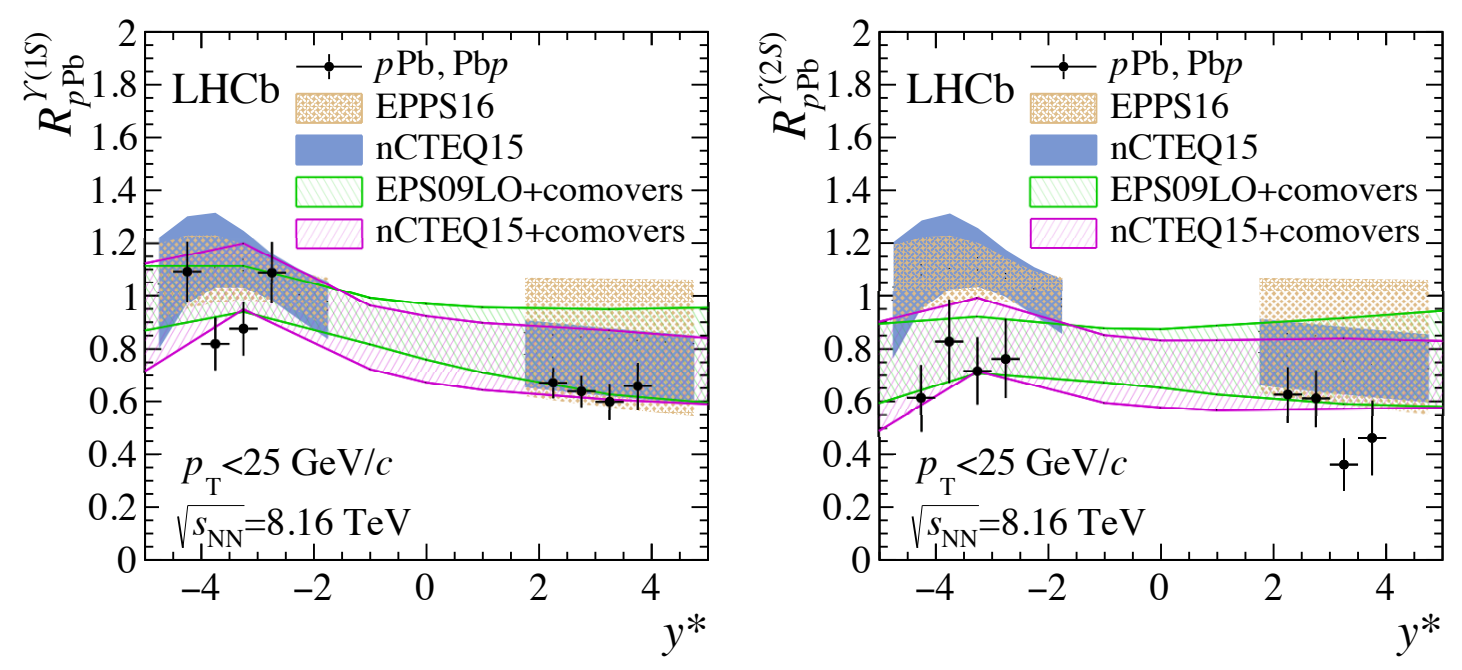

Figure 5: Nuclear modification factors of the $\Upsilon(1 \mathrm{~S})$ (left) and $\Upsilon(2 \mathrm{~S})$ (right) as a function of rapidity for the forward and backward samples. The results are compared with the theoretical predictions for the nCTEQ15

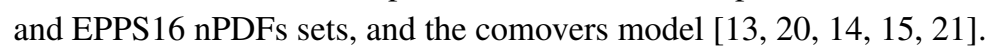


and $110.4 \mathrm{GeV}$ with fixed target configuration are shown in the Fig. [122]. The cross sections in $\mathrm{p}-\mathrm{He}$ collisions are compared to predictions [143, [15], 23]]. The predictions are scaled by a factor 1.78 in order to compare the shape of the distributions since total cross section is underestimated by the predictions. The differential yields with arbitrary normalization in $\mathrm{p}-\mathrm{Ar}$ collisions are compared to predictions and the $J / \psi$ results are also compared to interpolation from the results at the similar energy. As a result, no significant differences between data, predictions, and interpolations are observed. It suggests no evidence about strong intrinsic charm contribution within current uncertainties.
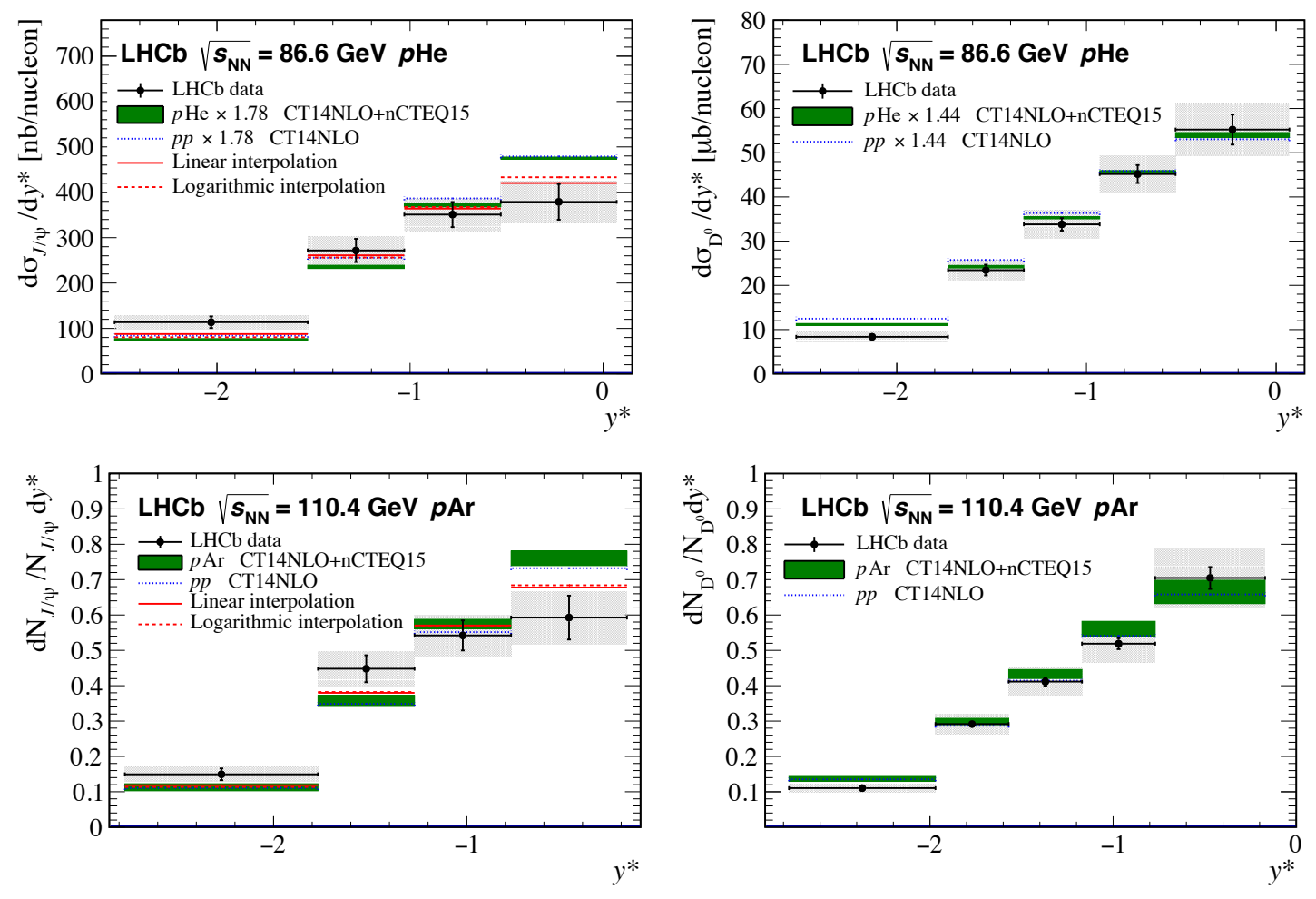

Figure 6: Differential $J / \psi$ production cross sections for $\mathrm{pHe}$ (top left) and differential $J / \psi$ yields for pAr (bottom left) collisions, and differential $D^{0}$ production cross sections for $\mathrm{pHe}$ (top right) and differential $D^{0}$ yields for pAr (bottom right) collisions, as a function of center-of-mass rapidity. The vertical lines express the quadratic sum of statistical and uncorrelated systematic uncertainties. The gray boxes express the correlated systematic uncertainties. The results are compared with HELAC-ONIA predictions [[13]].

\section{Summary}

Both ALICE and LHCb detectors have excellent capability of tracking and calorimetry. The measurements with these detectors enable complemental study using different detector coverage of rapidity. In heavy-ion collisions, hard probes are well calibrated probes of heavy-ion physics. Various measurements of hard probes have been carried out by the ALICE and LHCb collaborations at the LHC Run1 and Run2 experiments. Heavy-flavours production are studied by both experiments and it allows us to access wide rapidity range. In ALICE, jet production and jet 
substracture at mid rapidity range are studied by ALICE. In LHCb, fixed-target experiments using beam-gas interactions are carried out and it provides the unique opportunity to study nucleus-nucleus and proton-nucleus collisions on various targets. These heavy-flavour and jet measurements provide good tests for the $\mathrm{pQCD}$ and better understanding of the nuclear matter effects. During the current shutdown (LS2) of the LHC, both experiments are upgraded for the LHC Run3 [24, [25]. The physics capability in heavy-ion collisions will be enhanced by these upgrades and the further studies are promising.

\section{References}

[1] J. Collins, D. Soper, and G. Sterman, Nucl. Phys. B261 (1985) 104-142

[2] ALICE Collaboration, JINST 3 (2008) S08002

[3] LHCb Collaboration, JINST 3 (2008) S08005

[4] J. Mulligan on behalf of the ALICE Collaboration, PoS HardProbes2018 (2018) 080

[5] Y. He et al., Phys. Rev. C 99, 054911 (2019)

[6] A. Idilbi and A. Majumder, Phys. Rev. D 80 (2009) 054022

[7] G. Ovanesyan and I. Vitev, J. High Energy Phys. 06 (2011) 080

[8] J. Casalderrey-Solana et al., J. High Energy Phys. 10 (2014) 19

[9] K. C. Zapp, F. Krauss, and U. A. Wiedemann, J. High Energy Phys. 03 (2013) 080

[10] ALICE Collaboration, arXiv:1905.02512 [nucl-ex]

[11] ALICE Collaboration, Phys. Lett. B 793 (2019) 212

[12] LHCb Collaboration, JHEP 02 (2019) 102

[13] H. S. Shao, Comput. Phys. Commun. 198 (2016) 238

[14] K.J. Eskola, H. Paukkunen and C.A. Salgado, JHEP 04 (2009) 065

[15] K. Kovarik et al., Phys. Rev. D 93 (2016) 085037

[16] ALICE Collaboration, http://alice-collaboration.web.cern.ch

[17] X. Du, M. He, and R. Rapp, Phys. Rev. C 96 (2017) 054901

[18] P. P. Bhaduri et al., arXiv:1809.06235 [hep-ph]

[19] LHCb Collaboration, JHEP 11 (2018) 194

[20] E.G. Ferreiro, Phys. Lett. B 749 (2015) 98

[21] K.J. Eskola et al., Eur. Phys. J. C (2017) 77: 163

[22] LHCb Collaboration, Phys. Rev. Lett.122 (2019) 132002

[23] S. Dulat et al., Phys. Rev. D 93 (2016) 033006

[24] L. V. Palomo, J. Phys. Conf. Series 912, 1

[25] A. Piucci, J. Phys. Conf. Series 878, 1 